\title{
Highly Sensitive copper Heavy Metal Analysis on Nanoparticle Platinum and Palladium Electrode
}

\author{
Ahmad Khalaf Alkhawaldeh ${ }^{1,}{ }^{*}$, Rawia Alkhawaldeh ${ }^{1}$ \\ ${ }^{1}$ University of Jordan, Department of Chemistry, Amman - 11942, Jordan. \\ E-mail:Ahm9140096@fgs.ju.edu.jo,Ahmad.alkawalda@yahoo.com \\ Phone: 00962787455167
}

"Corresponding author: ahmad.alkawalda@yahoo.com

\begin{abstract}
The copper deposition on the platinum and palladium nanoelectrode has been studied using cyclic voltammetry. The use of nanoelectrode platinum and palladium are defined in the study of heavy metals. The noble nanoelectrode of metal has a typical silicone processing structure. In comparison to the nanoelectrodes, the geometry of the electrode series is complex and balanced. Nanoelectrodes of platinum are found effective in detecting heavy metal. There was regular analysis of the use of the sensors. The identification constraints down to the ng /L level was accomplished by refined electrode geometry and the stripping procedures. The process was used for the study of water sample determination. Another heavy metal ion attack voltammetric reaction was studied. The SEM picture clearly observed and characterized the nanoparticle electrode by X-ray diffraction and cyclic voltammetry.
\end{abstract}

Keywords: Platinum Electrode, Nanoparticle, Heavy metal, Square Wave Voltammetry.

\section{Introduction}

The general concern continues to track its exposure due to the well-documented adverse effects of heavy metals on the human body. In order to meet the requirements for wastewater or process management, heavy metal analysis is essential to industries (Lin and Huang, 2017). In comparison to atomically absorption or optical emission techniques, electrochemical stripping is a renowned method for heavy metals identification. The method of detection of the above provides extraordinary sensitivity. It can decide more than 30 elements (Hourani and Alkawaldeh, 2016). A hanged mercury drop (HMDE) or a thin-film of mercury (MFE) was widely used as an electrode in a supply (glass fiber, fiber paste). The HME is a thick electrode with a significant volume of radioactive mercury (Strmčnik et al, 2019). Both the MFE decreases the volume of mercury and the size of the electrode, but also requires a large amount. Nanoelectrodes were used for stripping processing, many methods are known (Altweiq and Alkhawaldeh, 2019).

Apart from nanoelectrode electrode electrodes, the amount of mercury used is slightly reduced due to the limited electrode surface (high current density, low signals to noise and hydrodynamic independence). One downside is that nanoelectrodes of this type are difficult to equip, where small $\mathrm{C}$, Ir, $\mathrm{Pt}, \mathrm{Au}$ or $\mathrm{Ag}$ fibers are closed into a nonconductor. This makes mass manufacturing immediately difficult (Cao et al, 2008). Photolithographic processing makes production more replicable and accurate. This article explains the properties of heavy metal detection nanoelectrode (Khedher et al, 2012).

In terms of diffusion properties, the effect of radius and distance between microelectrodes are discussed. Using cyclic voltammetry technique, nanoparticle platinum electrode materials were deposited. The creation of Mercury film is discussed and contrasts the electrode platinum to iridium. We addressed the reproducibility of the creation of platinum repeated mercury films and the repeat use of mercury films for study of Heavy Metal (Kokkinos et al, 2008). 
The diffusion function of the redox species to the electrode surface is an important distinction between macro- and nanoelectrodes. The cyclic voltammetry-metric answer is seen in different shapes. The diffusion function is planar for nanoelectrodes. A current peak is observed in the cyclic voltammetric reaction $(\mathrm{CV})$. The diffusion is hemispherical for microelectrodes. A constant voltammetric behavior can be seen in the current potentials map (Alkhawaldeh et al., 2020).

The platinum and palladium nanoparticle electrode study of copper $(\mathrm{Cu})$ is demon producing. There will be a question about the long-term durability of the heavy metal sensor.

\section{Experimental}

The chemicals used were of industrial quality and obtained by AldricWGermany, unless otherwise stated. With deionized and double distilled water, both solutions were made. For stock products, common methods have been used to evaluate AAS. The electrode was put in a $10 \mathrm{~mL}$ beaker of electrolyte solution during measurements. The nanoelectrodes were cycled three times in $0.1 \mathrm{M} \mathrm{HNO}_{3}$ between $\mathrm{E}=-0.6 \mathrm{~V}$ and $1.3 \mathrm{~V}$ in the first phase of use (sweep rate $\mathrm{u}=30 \mathrm{mV} / \mathrm{s}$ ). The solution was formed with a transmission of $\mathrm{NaSCN} 20.268 \mathrm{~g}, \mathrm{NHO}_{3} 20.01 \mathrm{~g}$ and $\mathrm{NH}^{+} 25 \%$ solution $18.78 \mathrm{~mL}$ into $1 \mathrm{~L}$ volume flask. Double distilled water has been added to the flask's top level. A blended Mercury AAS formula 1005pg / $\mathrm{mL} \mathrm{Hg}$ solution of $8.5 \mathrm{~mL}$ in 1 wt. percent $\mathrm{HNO} 03$ was combined with $1.5 \mathrm{~mL}$ of this solution. $\mathrm{As}, \mathrm{Pb}$, and $\mathrm{Cu}$ were found in 10 $\mathrm{mL}$ of a pH 4.5 acetate $\left(0.25 \mathrm{M}, \mathrm{HCH}_{3} \mathrm{CO}_{2}, \mathrm{NaCI}\right)$ normal buffer where sufficient metal content (10 to 90 $\mathrm{pL}$ of stock) has been applied.

A regular buffer acetate was developed in $1 \mathrm{~L}$ Volumetric Flask with a mixture of $2.2 \mathrm{~g}$ sodium acetate, $1.2 \mathrm{~mL}$ acetic acid and $14.6 \mathrm{~g} \mathrm{NaCl}$. The top mark of the flask was applied to double distilled water. $\mathrm{AS}, \mathrm{Pb}$, and $\mathrm{Cu}$ Stock Solutions have been prepared with the addition, in $10 \mathrm{~mL}$ from the acetate standard buffer, of 10PL of each AAS standard metal solution (1000 pg / mL).

The measurement of mercury was performed in $0.1 \mathrm{M} \mathrm{HCI} .10 \mathrm{ml}(10-40 \mathrm{pL})$ of an HCI solution of mercury stock $(100 \mathrm{pL})$ was given in $10 \mathrm{~mL}$ and $0.1 \mathrm{M}(\mathrm{HCl})$ by the $\mathrm{HCI}$ solution. Mercury was found in $0.1 \mathrm{M}$ HCI. In positioning procedures or stripping tests, oxygen elimination was not done. Even during the pre-concentration cycle were the electrolytes agitated.

\section{Results and Discussion}

Figure 1 shows SEM with various frequencies using platinum nanoelectrode, spherical and strong distribution of the nanoparticle in the air. A slight hysteresis is nevertheless apparent. This is consistent with a slight contribution from linear propagation before the state response is almost constant. Similar to the nanoelectrode, under the same laboratory conditions the current density $\mathrm{j}$ of the electrode is nearly 10 times greater. After a copper reoxidation in the solution, SEM images show that the coating is gradually raw at $0.8 \mathrm{~V}$ for $750 \mathrm{~s}$. Probably attributable to the amalgam coating of platinum and a partial platinum oxidation.

Figure 2 displays the platinum nano-particular electrode $\mathrm{CV}$ in a $0.5 \mathrm{M} \mathrm{KCl}$ solution. As predicted, a $\mathrm{CV}$ response for linear diffusion is seen in the nanoelectrode. The rotating heavy metal device can be used at its latest peaks. 


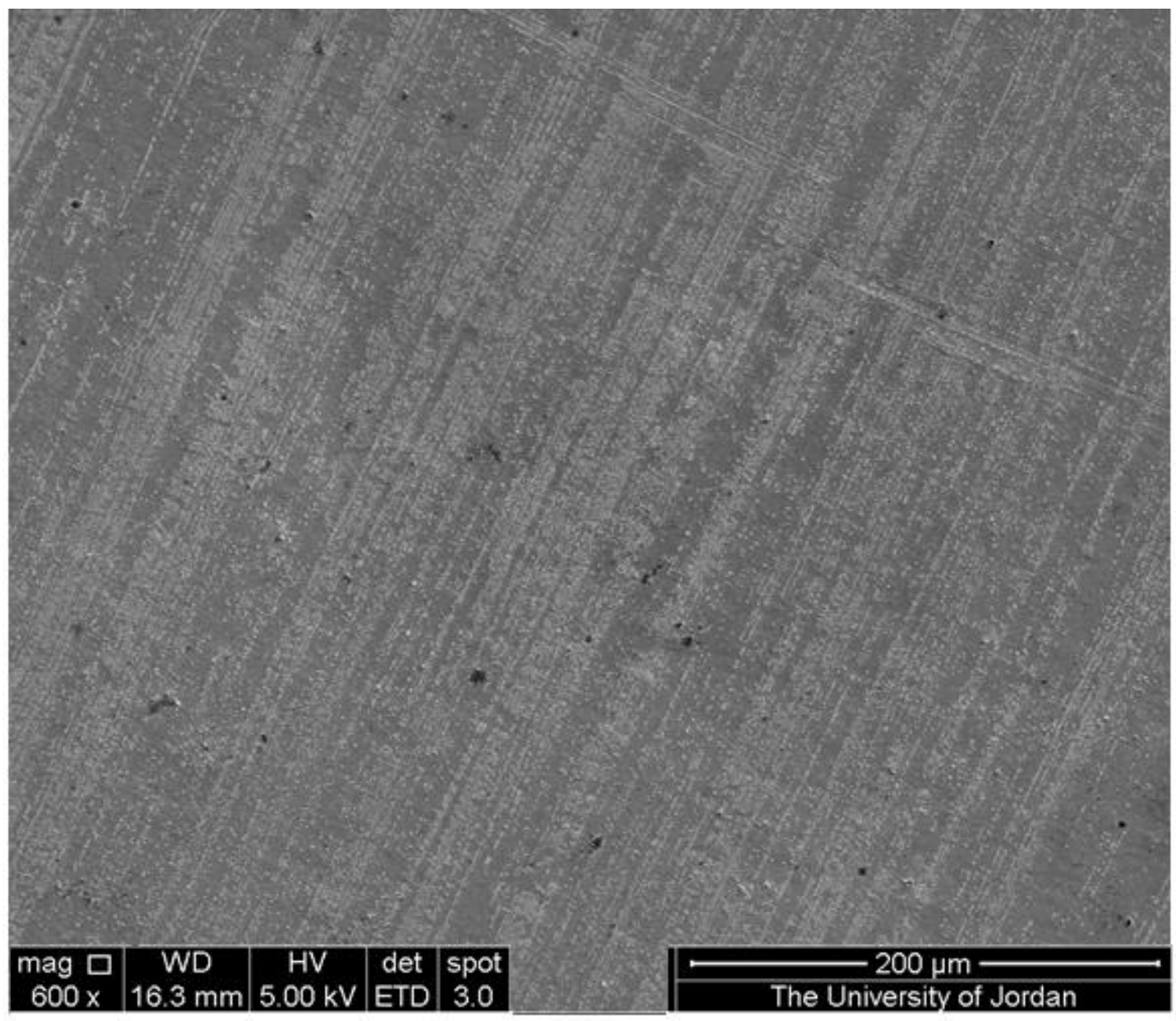

Figure 1. SEM picture of the platinum nanoelectrode.

In addition, the microelectrode array can apply square wave techniques with frequencies of up to $2000 \mathrm{~Hz}$ in comparison to usual analytical experience in nanoelectrodes. That is because the linear decay of the capacitive current into the electromagnetic radius is inversely proportional to the time constant. 


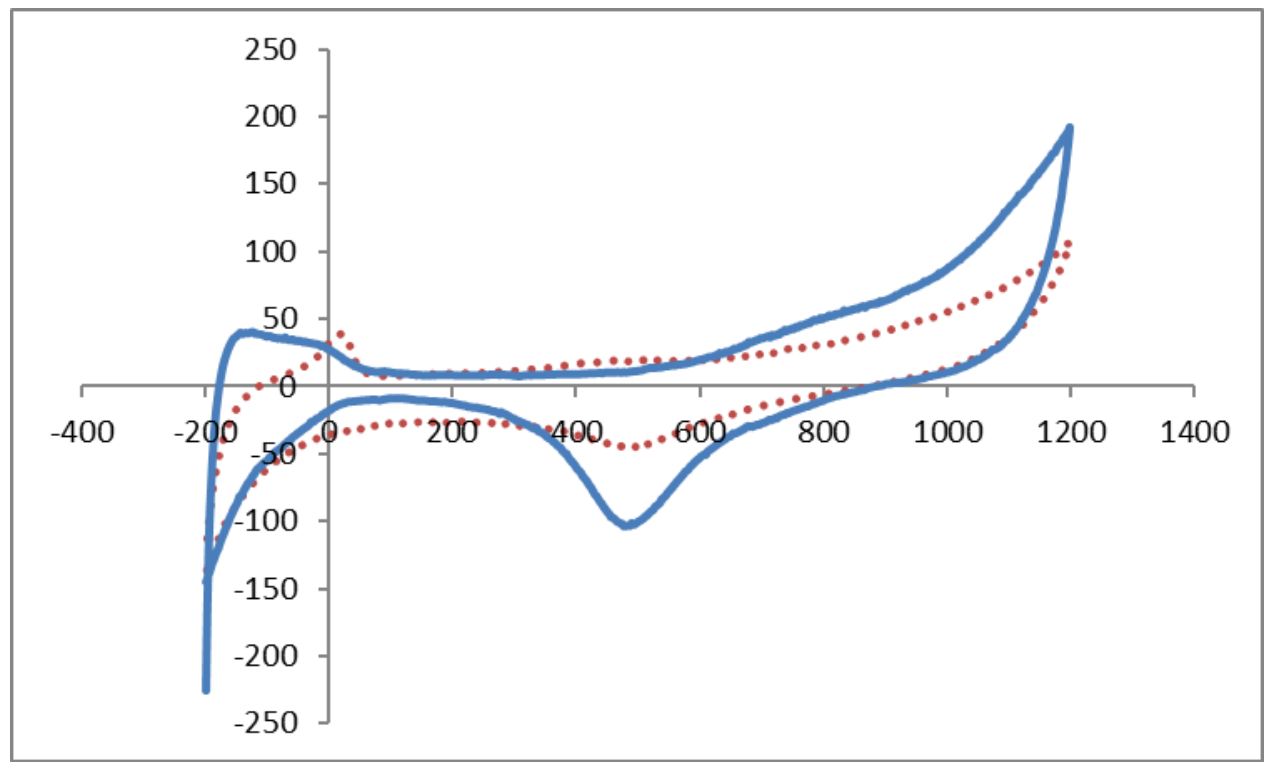

Figure 2. CV ofthe platinum nanoelectrode and copper heavy metal in $0.5 \mathrm{M} \mathrm{KCl}$

With rising square wave frequencies, the peak current and the peak area rise. It will greatly reduce the identification limit of the stripping study. The copper deposition was analyzed using cyclic voltammetry in the platinum nanoelectrode series. In Figure 3, a nanoelectrode in $\mathrm{Cu}^{2+}$ solution displays first consecutive cycles.

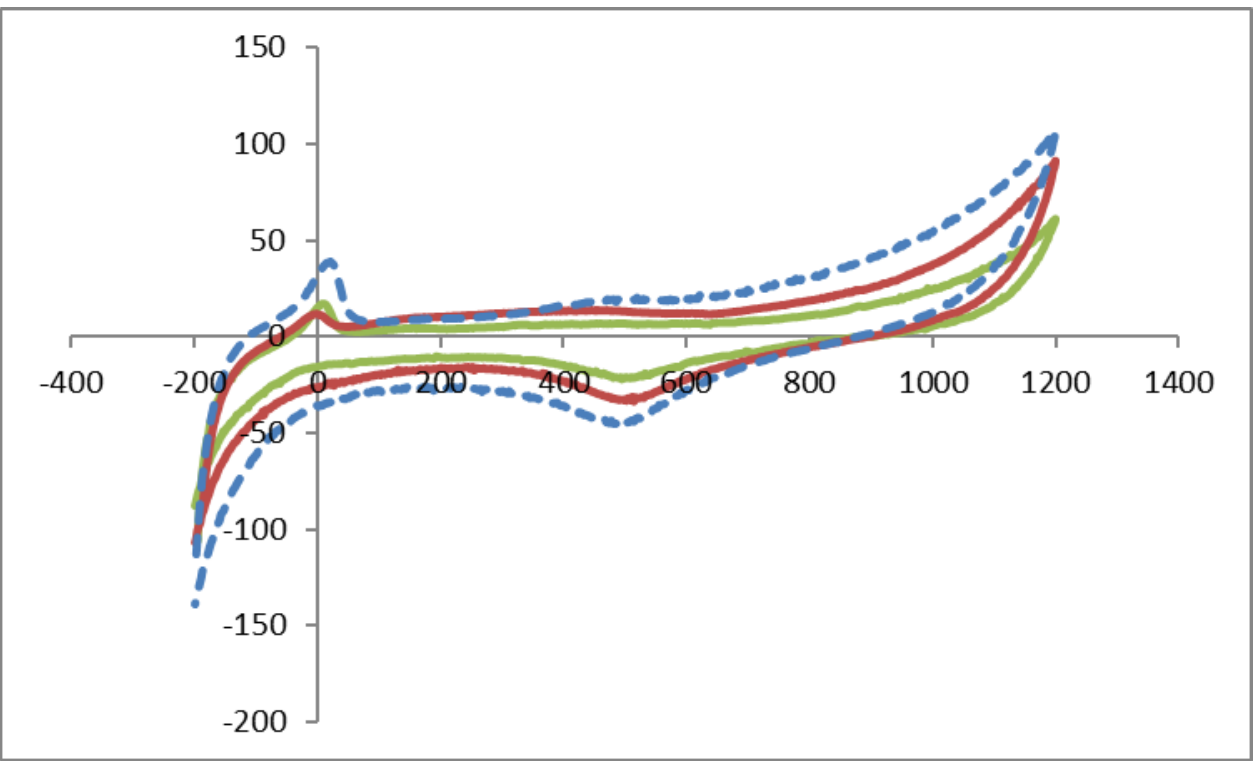

Figure 3. CV of copper heavy metal at different scan rate by the platinum nanoelectrode in $0.5 \mathrm{M} \mathrm{KCl}$.

The anodic current, as predicted for a nanoelectrode $\left(\mathrm{Cu}^{2+}\right.$ deposition), shows no current max. Following a transition of anodic direction in the first 5 scans, the half phase potential is stabilized. This is due to a change of the electrode surface during the palladium deposition. The copper coating is formed on 
the pure palladium surface during the first step. This mono-copper is not completely reoxidizable in the spectrum of potential added (Almatarneh et al., 2019).

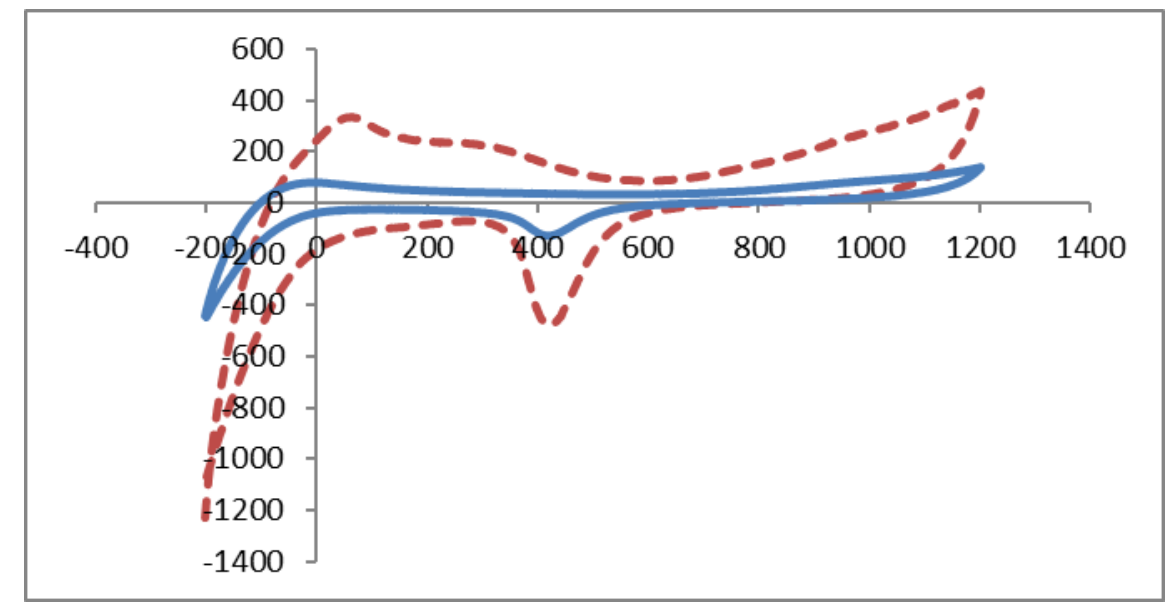

Figure 4. $\mathrm{CV}$ of the palladium nanoelectrode and copper heavy metal in $0.5 \mathrm{M} \mathrm{KCl}$.

A stable palladium nanoelectrode amalgam layer is seen in the difference in CV in Figure 4 in which the anodic $\left(\mathrm{Q}_{\mathrm{a}}^{+}\right)$or catholic $\left(\mathrm{Q}_{\mathrm{c}}^{-}\right)$charge is 4 . In subsequent cycles, the electrode surface is then created by a palladium amalgam. The copper deposition is also less over-potential (Laschi et al., 2006).

It is understood that platinum nanoelectrodes cannot produce a homogeneous copper ion. Copper droppings are distributed in homogeneously and do not overlap on the surface of the electrode).

One of the consequences is a high zero current due to the development of hydrogen in no amalgamated platinum. Moreover, platinum and palladium are co-adsorbed by metals that are to be observed to result in numerous cycles of oxidation (Alshamaileh et al., 2016). The copper content of the generated nanoelectrode and the microelectrode array was therefore evaluated with the zero current calculation in the anodic potential range.

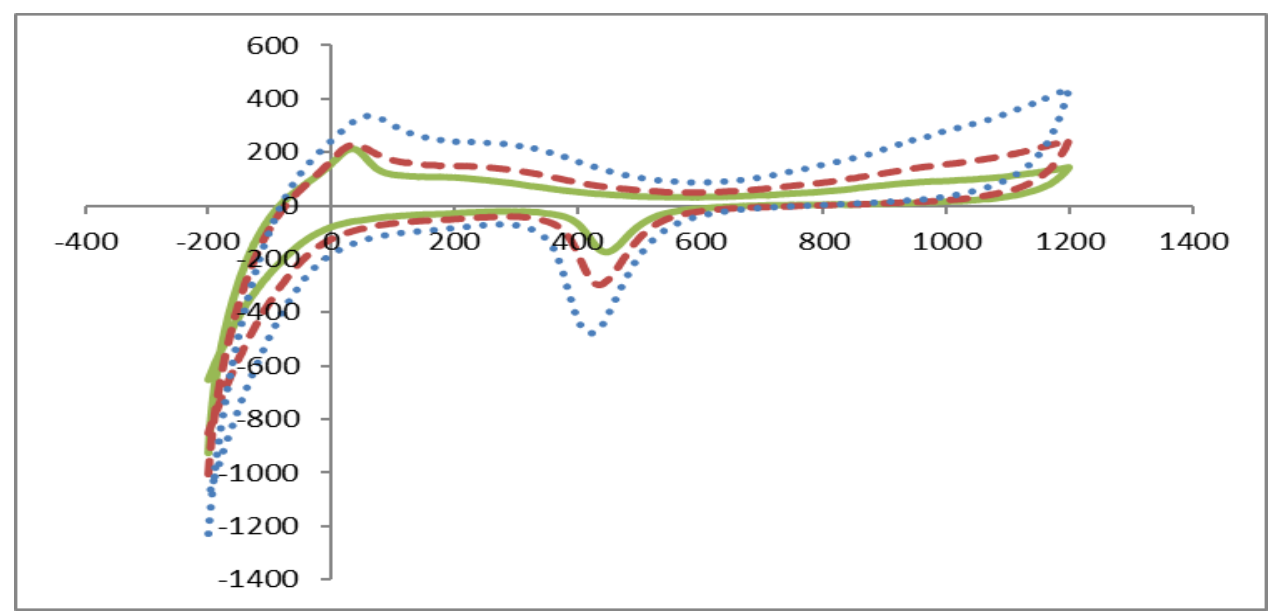

Figure 5. CV of copper heavy metal at different scan rate by the palladium nanoelectrode in $0.5 \mathrm{M}$ $\mathrm{KCl}$. 
The characterization took place in an acetate buffer solution that is later used for the identification of heavy metals. The nanoelectrode is null current compared with the electrode was slightly smaller. Based on the experimental conditions, a large current from the hydrogen production beginning at $-0.7 \mathrm{~V}$ was observed at the nanoelectrode, up to $-1.4 \mathrm{~V}$ a zero current of $2 \times 10^{-6} \mathrm{~A} / \mathrm{m}^{2}$ was reported (Krishan et al., 2019). Thus on the platinum nanoelectrode a homogeneous copper is formed. It seems that the "wall-like" structure of the insulating layer on top of the platinum disables the copper ion to spread out on the platinum surface. The consumed amount of copper for a formation on one nanoelectrode array is in the nomogram-range. This minimizes the risk of environmental pollution with copper during stripping analysis.

Copper will be inspected every day for corrosion in commercially available stripping inspection instruments. The copper coating must be reproducible with the reproductive properties of the subsequent plated copper ions in order to create a long-lasting heavy metal sensor. The reoxidation of the same geometrical nanoparticle platinum and palladium nanoelectrode was thus compared.

\section{Conclusions}

For highly sensitive heavy metal, copper analysis, platinum and palladium nanoelectrode arrays can be used. The nanoelectrodes are developed by standard IC technology and at a low price. The high mass flow observed allows an improved sample output relative to typical MFE in stripping experiments. In comparison, a higher faradic current to capacitive microelectrodes ratio enables square wave anodic stripping study with frequencies up to $2000 \mathrm{~Hz}$ compared with nanoelectrodes.

The consistency of the mercury coating on the platinum nanoelectrodes is small, unlike big platinum electrodes. The microelectrode's wall-like structure allows a homogenous cotton layer to be formed. In fact, the same platinum microelectrode series can be repeatedly revamped with a copper ion without loss of film consistency. Platinum can also be used as an electrode medium for stripping analysis. The application of platinum nanoelectrode enables the quick identification of heavy metals in the spectrum.

\section{References}

Alkhawaldeh, A. K., M.Krishan, M., Altwaiq, A., Dabaibeh, R. N. (2020). Preparation of Nanostructured/ Microplatinum Surfaces by Application of a Square Wave Potential Regime for Methanol Oxidation. Eurasian Journal of Analytical Chemistry, 15(1), emEJAC-00362.

Al-Khedher, M. A., Pezeshki, C., Mchale, J. L., \& Knorr, F. J. (2012). Empirical Modeling of Nanoindentation of Vertically Aligned Carbon Nanotube Turfs using Intelligent Systems. Fullerenes, Nanotubes and Carbon Nanostructures, 20(3), 200-215. doi: 10.1080/1536383x.2010.542590.

Almatarneh, M. H., Elayan, I. A., Al-Sulaibi, M., Khawaldeh, A., Saber, S. O. W., Al-Qaralleh, M., and Altarawneh, M. (2019). Unimolecular Decomposition Reactions of Propylamine and Protonated Propylamine. ACS Omega, 4(2), 3306-3313. doi: 10.1021/acsomega.8b02792.

Alshamaileh, E., Al-Sulaibi, M., Al-Khawaldeh, A., Almatarneh, M., El-Sabawi, D. and Al-Rawajfeh, A. (2016), "Current status of nanotechnology in Jordan", World Journal of Science, Technology and Sustainable Development, Vol. 13 No. 2, pp. 66-81. https://doi.org/10.1108/WJSTSD-01-2016-0001.

Altweiq, A. and Alkhawaldeh, A (2019). The Determination of Some Heavy Metals in Different Selected Diets. Eurasian Journal of Analytical Chemistry, 14(4), emEJAC-00326. 
Cao, L., Jia, J., \& Wang, Z. (2008). Sensitive determination of Cd and Pb by differential pulse stripping voltammetry with in situ bismuth-modified zeolite doped carbon paste electrodes. Electrochimica Acta, 53(5), 2177-2182. doi: 10.1016/j.electacta.2007.09.024.

Hourani, M. K. and Alkawaldeh A. (2016). Synergistic Effects of Bismuth Adatoms on Electrocatalytic Properties of Electrodeposited Nanostructured Platinum Electrodes. International Journal of Electrochemical Science, 3555-3566. doi: 10.20964/110434.

Kokkinos, C., Economou, A., Raptis, I., \& Efstathiou, C. E. (2008). Lithographically fabricated disposable bismuth-film electrodes for the trace determination of $\mathrm{Pb}(\mathrm{II})$ and $\mathrm{Cd}(\mathrm{II})$ by anodic stripping voltammetry. Electrochimica Acta, 53(16), 5294-5299. doi: 10.1016/j.electacta.2008.02.079.

Krishan, M.; Alkhawaldeh, A.; Soliman, A. (2019). Development of Nitride-Sensors for Monitoring in Control Systems. Preprints, 2019050227.

Laschi, S., Palchetti, I., \& Mascini, M. (2006). Gold-based screen-printed sensor for detection of trace lead. Sensors and Actuators B: Chemical, 114(1), 460-465. doi: 10.1016/j.snb.2005.05.028.

Lin, S.-L., \& Huang, W.-C. (2017). Chelation Therapy Has a Beneficial Effect in Ultrasonic Endothelial Function in Patients with Elevated Serum Lead or Cadmium Levels. Ultrasound in Medicine \& Biology, 43. doi: 10.1016/j.ultrasmedbio.2017.08.1930.

Strmčnik, E., Majdič, F., \& Kalin, M. (2019). Influence of a Diamond-Like Carbon-Coated Mechanical Part on the Operation of an Orbital Hydraulic Motor in Water. Metals, 9(4), 466. doi: $10.3390 /$ met9040466. 